\title{
Wissenschaftliche Begleitung und Versuchsschule: Was man aus der Evaluation schulischer Projekte über Schulentwicklung lernen kann
}

\section{Zusammenfassung}

In diesem Beitrag stellen wir das Konzept der wissenschaftichen Begleitung der HeleneLange-Schule, einer Versuchsschule des Landes Hessen, vor, illustrieren dieses anhand des Beispiels der wissenschaftlichen Evaluation und schulpraktischen Weiterentwicklung des Projekts „Jung \& Alt“ und ziehen aus den Erkenntnissen Schlussfolgerungen im Blick auf eine systemtheoretisch fundierte Theorie der Schulentwicklung. Wissenschaftiche Begleitung ist für uns eine "Win-win-Beziehung“, von der sowohl die Wissenschaft als auch die Schulpraxis profitieren. Sowohl das Thema Schulentwicklung (Kap. 3 und 6) als auch das Beispiel (Kap. 4 und 5) thematisieren wir jeweils aus wissenschaftlicher und schulischer Perspektive. Vorangestellt sind einige Erläuterungen zu den rechtlichen, organisatorischen und inhaltlichen Rahmenbedingungen, die sich aus dem Status der Helene-Lange-Schule als Versuchsschule des Landes Hessen ergeben (Kap. 1 und 2).

Schlüsselwörter: Versuchsschule, Universität, Zusammenarbeit, Schulentwicklung, Evaluation, Systemtheorie

\section{Cooperation of University and Laboratory School: Learning about School Improvement from Evaluating School Projects}

\section{Summary}

This article outlines the concept of the cooperation of the Department of Education at Goethe-University in Frankfurt and Helene-Lange-School in Wiesbaden. The article shows how school improvement, based on empirical research, can work, referring to the evaluation and further development of an extra-curricular school project ("Young \& Old"). The article concludes with a theoretical description of school improvement, based

1 Carmen Bietz verantwortet vor allem die Teile des Beitrags, die die schulische Perspektive darlegen (Kap. 1, 3 und 5), während Barbara Asbrand für die Beschreibung der qualitativrekonstruktiven Evaluationsforschung (Kap. 4) und die theoretische Einordnung (Kap. 6) zuständig war. An der Evaluation des außerunterrichtlichen Projektes, das in diesem Beitrag als Beispiel für das Konzept der wissenschaftlichen Begleitung dient, haben Dorthe Petersen und Susanne Thierolf $(\dagger)$ mitgearbeitet. 
on system theory, and explains how both school and university benefit from this cooperation.

Keywords: laboratory schools, university, cooperation, school improvement, evaluation, system theory

\section{Versuchsschule}

Die Helene-Lange-Schule, eine integrierte Gesamtschule in Wiesbaden, ist seit 1995 Versuchsschule des Landes Hessen. Eine Versuchsschule dient „der Weiterentwicklung des Schulwesens durch Erprobung von Veränderungen und Ergänzungen in Didaktik, Methodik und Aufbau einer Schule“ (Hessisches Schulgesetz, \$14). Versuchsschulen sind auf Dauer angelegt, übernehmen ähnliche Aufgaben wie „Entwicklungsabteilungen“" in Betrieben und sind im Gegensatz zu Schulversuchen nicht befristet. Was in den Versuchsschulen entwickelt wird, wird in Erlassen des Hessischen Kultusministeriums definiert; es muss durch die Schule dokumentiert, veröffentlicht und evaluiert werden. Die Schule muss sich Besucherinnen öffnen und diesen die Möglichkeit geben, Ideen, Materialien und Konzeptbausteine auf ihre eigene Situation zu übertragen. In Revisionsgesprächen, an denen Vertreter*innen des Kultusministeriums, des Staatlichen Schulamtes, des Schulträgers und der Schulgemeinde teilnehmen, erläutern Schulleitung und Kollegium der Helene-LangeSchule, welche Fortschritte und Erkenntnisse bezüglich der Versuchsschulaufträge gemacht und wie die entsprechenden Ressourcen eingesetzt wurden.

Der aktuelle Versuchsschulerlass sieht vor, dass die Helene-Lange-Schule in eine „Selbstständige Schule“ umgewandelt ist und, als eine der Schulen im zweiten Jahrgang dieser Umwandlung, insbesondere Erfahrungen mit den Möglichkeiten des "Großen Schulbudgets" und dem Aufbau eines Qualitätsmanagementsystems sammeln und evaluieren soll. Dabei gilt es, die besondere pädagogische Prägung der Schule mit weiterzuentwickeln. Außerdem nimmt die Helene-Lange-Schule im Zusammenhang mit dem nun in Hessen auf Antrag möglichen Verzicht auf äußere Fachleistungsdifferenzierung in Integrierten Gesamtschulen besondere Aufgaben wahr. Die Schule bringt ihre langjährigen Erfahrungen mit binnendifferenziertem Unterricht in verschiedenen Zusammenhängen ein; u. a. war sie gemeinsam mit den anderen hessischen Versuchsschulen an einer Arbeitsgruppe des Hessischen Kultusministeriums zum Thema „Verzicht auf äußere Fachleistungsdifferenzierung“ beteiligt. Gemeinsam mit den anderen hessischen Versuchsschulen - der Offenen Schule Kassel-Waldau, der Reformschule Kassel und der Steinwaldschule Neukirchen - wird ein jährlich stattfindender Fachtag „Binnendifferenzierung“ organisiert. Die HeleneLange-Schule berät Schulen auf Anfrage zum vollständig binnendifferenzierten Unterricht und gibt ihre Erfahrungen weiter. 
Konzeptionelle Weiterentwicklung geschieht, wie im Versuchsschul-Erlass formuliert, in folgenden Themenbereichen:

- schülerzentrierte und kompetenzorientierte Lernformen (insbesondere „Selbständiges Lernen“ und systematische Nutzung außerunterrichtlicher Lernorte);

- Arbeit mit Diagnoseinstrumenten, insbesondere mit Kompetenzrastern und Kompetenzprofilen;

- Entwicklung eines kompetenzorientierten Medienkonzepts;

- Zusammenarbeit mit der Schule für Erziehungshilfe und dem Beratungs- und Förderzentrum (BFZ) in der inklusiven Beschulung;

- gezielte, individuelle Förderung hochbegabter Schülerinnen;

- Weiterentwicklung der Bewertung in Form von Lernentwicklungsberichten.

Die Wirkung der Helene-Lange-Schule resultiert zum einen aus ihrer Modellfunktion und zum anderen aus der Weitergabe der Erkenntnisse bezüglich der Gelingensbedingungen von Schulentwicklungsprozessen und Konzeptbausteinen. Zahlreiche Veröffentlichungen, Fortbildungsangebote, Hospitationsprogramme und die Mitarbeit in Netzwerken sorgen dafür, dass die Erfahrungen weitergegeben werden. Beispielsweise das Teamprinzip und die Jahresarbeitspläne wurden von vielen Schulen übernommen.

\section{Wissenschaftliche Begleitung}

Die wissenschaftliche Begleitung, die gemäß der Regelungen des Hessischen Schulgesetzes für die Versuchsschulen verbindlich ist (HSchG $\$ 14$ ), geschieht in Zusammenarbeit mit dem Fachbereich Erziehungswissenschaften der Goethe-Universität Frankfurt am Main. Seit einigen Jahren übernehmen Prof. Dr. Barbara Asbrand und ihre Arbeitsgruppe die wissenschaftliche Begleitung der Helene-Lange-Schule; allerdings ist diese weder innerhalb der Universität institutionell abgesichert noch wird sie durch zusätzliche finanzielle Mittel des Landes unterstützt. Vielmehr basiert die Zusammenarbeit auf der Verabredung, dass beide Partner die Kooperation jeweils gewinnbringend für sich nutzen können, und auf einem beiderseitig geteilten Interesse an Zukunftsthemen der Schule. Universitätsseitig findet das Engagement der Professorin und der Mitarbeiter*innen in der Begleitung der Schulpraxis im Rahmen der universitären Aufgabe des Wissenschaftstransfers statt. Dass dies in größerem Umfang möglich ist, ist auch der Tatsache geschuldet, dass seitens der GoetheUniversität dem Dialog mit der Praxis und dem Transfer wissenschaftlichen Wissens in die Praxis als dritte Säule neben den zentralen universitären Aufgaben der Lehre und der Forschung seit einigen Jahren vermehrt Bedeutung beigemessen wird. Aus schulischer Perspektive ist von Bedeutung, dass der von der wissenschaftlichen Begleitung gewählte Forschungszugang als anschlussfähig an die schulische Praxis eingeschätzt wird. Das genaue Beobachten, Zuhören, Nachfragen und Gespräche- 
Führen mit Schüler*innen und allen an Prozessen Beteiligten im Rahmen der dokumentarischen Evaluations- bzw. Unterrichtsforschung passt zur schulischen Praxis, bei der Schülerorientierung im Fokus steht. Eine Forschungsperspektive, deren Ziel es ist, Bedingungen für in die Tiefe gehendes Lernen zu rekonstruieren, ist für die schulischen Praktiker*innen nicht nur akzeptabel, sondern auch gewinnbringend und zielführend.

Zusammengefasst lässt sich festhalten: Unsere Zusammenarbeit von Versuchsschule und Universität ist - anders als im Fall von Universitätsschulen - nicht institutionalisiert, sondern wird in Abhängigkeit von der inhaltlichen und forschungsmethodischen Ausrichtung immer wieder neu ausgehandelt. In den folgenden Kapiteln erläutern wir anhand eines Beispiels, wie sich die o.g. Passungsverhältnisse von Schulentwicklung und wissenschaftlicher Begleitung in unserer Schul- und Forschungspraxis darstellen.

\section{Schulentwicklung der Helene-Lange-Schule}

Die Versuchsschulaufträge sind Teil eines komplexen Zielsystems für die Schulentwicklung der Helene-Lange-Schule: Neben den Zielen als Versuchsschule gibt es Ziele als Selbstständige Schule, aus den Zielgesprächen zur Schulinspektion, aus den Netzwerken der Schule und natürlich auch aus dem Kollegium. In einem aufwändigen mehrschrittigen Prozess wurden die vielen Einzelziele sinnvoll zusammengefasst und werden nun in einigen wenigen, durch das Kollegium priorisierten Schulentwicklungs-Arbeitsgruppen bearbeitet. Diese arbeiten gegenwärtig zu den Themen „Ganztag“, „Medienkompetenz-Entwicklung“, „Inklusion“, „Kompetenzorientierung" und „Lernen an außerschulischen Orten“. Inhaltlich werden die Arbeitsgruppen durch die Planungs- und Steuerungsgruppe und themenbezogene Zusammenarbeit verzahnt. Sie erhalten ihre Aufträge von der Gesamtkonferenz und sind der Schulleitung und der Gesamtkonferenz rechenschaftspflichtig.

Das Schulentwicklungs-Thema "Lernen an außerschulischen Orten“ ist mit hohen pädagogischen Zielen verbunden und findet sich an der Helene-Lange-Schule in allen Jahrgängen in verschiedenen Ausprägungen (vom fünfwöchigen Theaterprojekt bis zum Sozialpraktikum) wieder. Ziele der außerunterrichtlichen Projekte liegen hauptsächlich beim Erwerb wichtiger Schlüsselkompetenzen durch Verantwortungsübernahme (für sich selbst und für andere), bei Rollen- und Selbstfindung, intensiver Auseinandersetzung mit einer Erfahrung oder einem Gegenstand ohne schulische Zwänge, Annehmen und Meistern von Herausforderungen, Engagement für andere. Jugendliche Schüler*innen wollen oft nicht mehr „so tun als ob“, sondern wirklich handeln und sich erproben im „echten“ Leben - sicher mit ein Grund dafür, dass Lernen im Fachunterricht in der Pubertät oft schwierig ist. Umso bereichernder 
wird die Erfahrung wahrgenommen, dass Jugendliche außerhalb von Schule motiviert und engagiert bei der Sache und bei sich selbst sind.

Die Arbeitsgruppe $\mathrm{zu}$ außerunterrichtlichen Lernorten hat sich mit verschiedenen Entschulungs-Modellen beschäftigt und ist in Bezug auf die Umsetzbarkeit neuer Konzepte, aber auch was Fragen der Zielerreichung angeht, an Grenzen gestoßen. Gleichzeitig wird das Lernen außerhalb von Stundenplänen und Unterrichtsräumen von allen Beteiligten als große Chance angesehen. Im Rahmen des Schulentwicklungsprozesses sollten deshalb die bestehenden außerunterrichtlichen Projekte evaluiert und dabei insbesondere die Passung von Methoden und Zielen überprüft werden. Die wissenschaftliche Begleitung wurde zur Teilnahme und Mitarbeit in der Schulentwicklungs-Arbeitsgruppe eingeladen und übernahm den Evaluationsauftrag der Schule. Ziel dabei war es, systematisch zu erheben, welche Erfahrungen die Jugendlichen in den Projekten machen, was sie als Herausforderung erleben und welche Kompetenzen sie erwerben.

Wie wir die wissenschaftliche Begleitforschung praktizieren, werden wir im Folgenden beispielhaft anhand der Evaluation und Weiterentwicklung des Projekts „Jung \& Alt" darstellen. Dieses Projekt wird seit vielen Jahren durchgeführt und findet im Jahrgang 8 statt. Anstelle von Religionsunterricht in der Schule besuchen die Schüler*innen einen älteren Menschen und reflektieren ihre Erfahrungen bei einer Auswertungsfahrt ins Kloster. Erhofft hat sich die Schule von diesem Projekt Verantwortungsübernahme, Hilfe für den Senior oder die Seniorin („tätige Nächstenliebe“ war der ursprüngliche Name des Projekts), echte persönliche Begegnungen mit älteren Menschen, das Erleben von Toleranz und Respekt, Nachdenken über Alter und Tod. „Bauchweh“ hatten die Kolleg^innen bei dem Namen „tätige Nächstenliebe“ und den hohen Zielen. Es stand an zu prüfen, was genau Schüler*innen bei diesem Projekt lernen (können) und inwieweit es tatsächlich dazu geeignet ist, Verantwortungsübernahme zu erlernen.

\section{Wissenschaftliche Begleitung als dokumentarische Evaluationsforschung}

Für die Evaluation der außerunterrichtlichen Projekte wurden im Rahmen eines Lehrforschungsprojekts von Masterstudierenden der Erziehungswissenschaft je Projekt mehrere Gruppendiskussionen mit Schüler ${ }^{\star}$ innen durchgeführt. Die Jugendlichen wurden aufgefordert, von ihren Erlebnissen in den Projekten zu erzählen; ausgewertet wurden die Gruppendiskussionen mit der Dokumentarischen Methode (Bohnsack, 2014). ${ }^{2}$ Neben den verschiedenen außerunterrichtlichen Projekten der

2 An den Interpretationen, aus denen die Evaluationsergebnisse hervorgegangen sind, haben neben den Studierenden auch die Wissenschaftlerinnen maßgeblich mitgewirkt. 
Helene-Lange-Schule, die untereinander vergleichend analysiert wurden, wurden auch Gruppendiskussionen, die mit Teilnehmenden von Theaterprojekten und Entschulungsprojekten an anderen Schulen und in außerschulischen Kontexten durchgeführt und zum Teil im Rahmen von Masterarbeiten erhoben wurden, in die komparative Analyse einbezogen.

Hieran ist die Konzeption der wissenschaftlichen Begleitung der Helene-LangeSchule als „Win-win-Situation“ zu erkennen: Die schulischen Akteure erhalten aus der Datenerhebung und -analyse eine Rückmeldung, die der Weiterentwicklung ihrer Praxis dient, während die Wissenschaftler`innen die Daten für Forschungsprojekte nutzen und aus praxisnahen Fragestellungen Forschungsthemen generieren, die auch in wissenschaftlicher Hinsicht von Interesse sind (vgl. Rosenberger, in Vorb.). Methodologisch orientiert sich diese Forschung am Konzept der dokumentarischen Evaluationsforschung (vgl. Bohnsack, 2006; Bohnsack \& Nentwig-Gesemann, 2010; Lamprecht, 2012). Dies macht es möglich, die auf die Weiterentwicklung der schulischen Praxis ausgerichtete Evaluation und die erziehungswissenschaftliche Grundlagenforschung miteinander zu verknüpfen. Denn die dokumentarische Evaluationsforschung zeichnet sich u. a. dadurch aus, sich auch in Evaluationsprojekten an den Qualitätsstandards der qualitativ-rekonstruktiven Sozialforschung zu orientieren (vgl. Bohnsack, 2006). Die Verknüpfung der Evaluation der schulischen Praxis mit Vorhaben der Grundlagenforschung ist die Voraussetzung dafür, dass die wissenschaftliche Begleitung auch für die Forscher*innen der Universität einen „Win-Faktor“ beinhaltet.

Des Weiteren sind grundlegende Prinzipien der Dokumentarischen Methode von Bedeutung; zu nennen sind hier insbesondere die Leitdifferenz von kommunikativem bzw. explizitem und konjunktivem bzw. implizitem Wissen im Anschluss an Mannheims Wissenssoziologie (Mannheim, 1980) und die Einklammerung des Geltungscharakters. Das Potenzial der dokumentarischen Methode, welches sich auch für Evaluationen als sehr nützlich erwiesen hat, liegt in der Möglichkeit, implizites Wissen empirisch rekonstruieren zu können, welches das Alltagshandeln bestimmt, in der Regel aber in der Handlungssituation nicht reflexiv zugänglich ist. Im Kontext der Evaluationsforschung wird der Mannheimschen Unterscheidung zwischen konjunktivem und kommunikativem Wissen folgend zwischen expliziten Bewertungen und impliziten Werthaltungen unterschieden (vgl. Bohnsack, 2006). Die Evaluation soll dazu führen, die expliziten Bewertungen und Ziele der Evaluierten mit den rekonstruierten impliziten Wissensbeständen und Praktiken zu relationieren und letztere der Reflexion, der Bewertung und der Veränderung zugänglich zu machen (ebd.). Das Prinzip der „Einklammerung des Geltungscharakters“ (Mannheim, 1980, S. 88) bedeutet, kurz gesagt, als Wissenschaftler*in kein „Besserwissen“ zu beanspruchen, sondern auch forschungspraktisch die erkenntnistheoretische Prämisse umzusetzen, dass Wissenschaft über keine bessere Erkenntnis verfügt als die Praxis, sondern im Modus der Beobachtung zweiter Ordnung eine andere Perspektive auf die 
Praxis einnimmt. Eingelöst wird dieser Anspruch durch den Rekurs auf empirische Vergleichshorizonte und den Verzicht auf Interpretationen auf der Basis der standortgebundenen Perspektiven der Forscher ${ }^{\star}$ innen (vgl. Bohnsack, 2014, S. 65 ff., 191 ff.). Im Kontext der Evaluationsforschung kommt als drittes grundlegendes Prinzip das der Responsivität hinzu. Damit ist bezeichnet, dass Schlussfolgerungen aus den Rekonstruktionen nicht von den Wissenschaftler ${ }^{*}$ innen formuliert, sondern im kommunikativen Austausch mit der Praxis entwickelt werden (vgl. Lamprecht, 2012).

Auf dieser Grundlage wurden die Erfahrungen der Schüler ${ }^{\star}$ innen in den außerunterrichtlichen Projekten auf der Ebene des konjunktiven Wissens rekonstruiert und mit den expliziten Zielen der Schule relationiert. Im Fall des Projekts „Jung \& Alt“ bestanden die zentralen Befunde darin,

- dass die Strukturen der Kontaktaufnahme mit den Senior ${ }^{\star}$ innen uneindeutig sind, da die Ziele des Projekts unklar sind,

- dass die älteren Menschen und die Schüler*innen unterschiedliche Auffassungen von der Dauer und der Qualität ihrer Beziehung haben, was von den Jugendlichen teilweise als belastend erlebt wird, und

- dass die Lernerfahrungen der Schüler*innen im Rahmen des intergenerationellen Lernens eher Themen des Faches Gesellschaftslehre als die implizit karitative Ausrichtung des Projekts betreffen.

Diese Ergebnisse wurden seitens der Wissenschaftlerinnen der Fachkonferenz Religion zurückgemeldet und bei einem Treffen der Schulentwicklungs-Arbeitsgruppe „Lernen an außerschulischen Orten“, an dem auch Mitglieder der Schulleitung teilnahmen, zur Diskussion gestellt.

\section{Schulpraxis: Wie sich das Projekt „Jung \& Alt“ im Anschluss an die Evaluation verändert hat}

Die Fachsprecherin Religion, auf deren Erfahrungsbericht die folgenden Ausführungen beruhen, erhielt erste Hinweise über Evaluationsergebnisse über den Beauftragten für Qualitätsmanagement der Schule, der sich intensiv mit den Ergebnissen der Evaluation der außerschulischen Lernorte durch die wissenschaftliche Begleitung beschäftigt hatte und erste Eindrücke gezielt an die in der Schule Handelnden weitergab. Diese erste Rückmeldung wurde als Irritation wahrgenommen: Heißt das jetzt, dass wir das Projekt kippen sollen? Passt es überhaupt in den Religionsunterricht? Ist das ganze Projekt fragwürdig? Nachdem die wissenschaftliche Begleitung in der Fachkonferenz von den Ergebnissen der Evaluation berichtet hatte, haben zwei wesentliche Erkenntnisse die Kolleg*innen veranlasst, das Projekt beizubehalten, aber deutlich zu überarbeiten. Erstens: Das Projekt wirkt inhaltlich und von den Anforderungen an die Jugendlichen her überfrachtet (z. B. was das Finden einer Projekt-Partnerin bzw. eines Projekt-Partners angeht) und von den Zielen her unklar; 
zweitens: „Tätige Nächstenliebe“ passt nicht zum Abbruch der Beziehung zwischen den Schüler*innen und ihrer "Reli-Oma“ bzw. ihrem "Reli-Opa“ nach Beendigung des Projektes.

Die Kolleg*innen haben auf der Grundlage der Evaluationsergebnisse entschieden, von nun ab den Fokus auf die intergenerationelle Kommunikation (und nicht mehr auf das Engagement für andere) zu legen. Dazu wurde ein Kompetenzraster entwickelt, das Selbst- und Fremdeinschätzung sowie Zieltransparenz ermöglicht. Außerdem gibt es nun ein Anfangs- und ein Abschlusstreffen aller Beteiligten, bei dem Ziele des Projektes erläutert werden und die Schule sich bei den älteren Menschen für ihr Ehrenamt bedankt. Alle Beteiligten werden zu einem Kaffeeklatsch mit kleinem Rahmenprogramm eingeladen; so werden sie in das Projekt eingeführt und können es auch gemeinsam abschließen. Die Kolleg*innen führen eine Kartei mit den Kontaktdaten älterer Menschen, die gern am Projekt teilnehmen möchten und auf die die Jugendlichen zurückgreifen können.

Dass die positive Entwicklung anhält und Veränderungen nachhaltig werden, erfordert weitere Maßnahmen. So werden beispielsweise regelmäßig auf der schulinternen Übergabekonferenz der Jahrgangsteams Materialien und Erkenntnisse weitergegeben. Die Fachsprecherin Religion kümmert sich darum, dass das entsprechende Jahrgangsteam das Projekt rechtzeitig in den Blick nimmt; dabei übergibt sie einen nach den Erfahrungen des letzten Jahrgangs aktualisierten „Fahrplan“.

Die Weiterentwicklung des Projektes „Jung \& Alt“ wird in der Schule positiv gesehen. Durch die konstruktive und auf den Punkt gebrachte Kritik war es den Kolleg*innen möglich, konkrete Veränderungen vorzunehmen, so dass nun eine höhere Passung von Ziel und Methode erlebt wird und alle sicher sind, dass das Projekt so, wie es jetzt ist, ein wichtiger Bestandteil des Kompetenzerwerbs im Rahmen des Religionsunterrichtes ist. Dass dazu der unterrichtliche Rahmen gesprengt wird, wirkt auf die Beteiligten in der Neukonzeption passend und zielführend. Die Kolleg*innen erleben sowohl die Überarbeitung des bestehenden Konzeptes in Auseinandersetzung mit Evaluationsergebnissen als auch die anschließende Durchführung als freudvoll und schätzen nicht nur die klare, andere Perspektive der wissenschaftlichen Begleitung, sondern auch das spürbare persönliche Interesse und Engagement der Professorin an ihrem schulischen Thema. Eine erneute Evaluation des veränderten Projektes halten die in der Schule Handelnden für sinnvoll, um sich zu vergewissern, dass man mit den angestoßenen Veränderungen auf dem passenden Weg ist. 


\section{Schulentwicklung als evolutionärer Prozess}

Für das Verständnis des Funktionierens des Zusammenspiels von wissenschaftlicher Begleitung und Schulentwicklung an der Versuchsschule ist ein systemtheoretisches Verständnis von Schulentwicklung hilfreich. Vorausgeschickt sei, dass wir mit dem Projekt „Jung \& Alt“ bewusst ein Beispiel eines erfolgreichen Entwicklungsprozesses gewählt haben, anhand dessen sich einige Gelingensbedingungen der von uns praktizierten Form der Zusammenarbeit von Wissenschaft und Schulpraxis aufzeigen lassen. Selbstverständlich könnten wir aus mehreren Jahren der Zusammenarbeit auch zahlreiche Beispiele berichten, in denen die Rückmeldungen von Evaluationsergebnissen in der Schulpraxis nicht anschlussfähig waren und im Sande verlaufen sind. Wird Schulentwicklung als sozialer Wandel auf der Basis evolutionärer Prozesse aufgefasst, lässt sich beides theoretisch einordnen, ihr Gelingen und ihr Scheitern.

Emmerich und Maag Merki (2014) beschreiben Schulentwicklung als Strukturveränderung der Einzelschule in Auseinandersetzung mit dem gesellschaftlichen Wandel in der Umwelt der Schule und unterscheiden dabei zwischen Evolution und Entwicklung. Während letztere ein durch verschiedene Akteure geplantes und zielgerichtetes Vorgehen bezeichnet, beschreibt der Begriff der Evolution Prozesse des Wandels, die allerdings nur ex-post als solche erkannt werden können. Während Emmerich und Maag Merki zwar Schule als soziales autopoietisches System auffassen, sehen sie den Gegenstand der Schulentwicklungsforschung bzw. -theorie dennoch bei den geplanten und zielgerichteten Entwicklungsprozessen (ebd., S. 2 f.). John (2013) dagegen denkt sozialen und organisationalen Wandel konsequent als soziale Evolution im Sinne Luhmanns (1998, S. 413 ff.). John definiert Innovationen als etwas Neues, Andersartiges und Abweichendes, das sich als Problemlösung bewährt hat und im Rückblick als relevante Neuerung bewertet wird. Während Planungen auf der Basis von Entscheidungen in Organisationen prozessiert werden und immer der Gefahr des Scheiterns ausgesetzt sind, was mit Hilfe von Innovations-, Fortschritts- und Reformsemantiken abgemildert wird, werden Innovationen als Ergebnis der sozialen Evolution aufgefasst und sind qua Definition mit Erfolg verbunden, da die Bewertung einer Veränderung als Innovation mit der nachträglichen Markierung als relevanter, bewährter Problemlösung einhergeht (vgl. John, 2013, S. $79 \mathrm{ff}$.). Der evolutionäre Veränderungsprozess umfasst Irritationen als auslösende Momente, Variationen des Strukturerhalts (womit gleichzeitig bestehende Strukturen in Frage gestellt werden), die Selektion einer der Alternativen (und das gleichzeitige Scheitern aller anderen) sowie ihre Restabilisierung. Innovation ist demnach das „jeweils Fortgeführte, das nicht irrelevant, abgelehnt oder zurückgewiesen wurde“ (ebd., S. 81).

Anhand des oben geschilderten Beispiels aus der Praxis der wissenschaftlich begleiteten Schulentwicklung lässt sich nun aufzeigen, unter welchen Bedingungen solche 
evolutionären Prozesse des Wandels innerhalb der Organisation Schule möglich werden:

(1) Irritation: Ein wichtiges Prinzip der wissenschaftlichen Begleitung der HeleneLange-Schule ist die Praxis, dass die Forschungsgegenstände durch die schulischen Akteure definiert werden. Das bedeutet, die Forschung beschäftigt sich mit Fragestellungen, die in der Schulpraxis als Problem identifiziert wurden und dort nach einer Problemlösung verlangen. Es sind nicht die empirisch forschenden Wissenschaftlerinnen, die daten- bzw. evidenzbasiert Problembereiche der schulischen Praxis identifizieren und als eine Intervention von Außen eine Irritation des schulischen Systems erzeugen. Vielmehr entsteht die Irritation im System der Praxis. ${ }^{3}$ Im konkreten Fall bemerken die Lehrpersonen ein Unbehagen in der Durchführung der außerunterrichtlichen Projekte; insbesondere die Unklarheit und der hohe Anspruch der Ziele bereiten „Bauchweh“ (s.o.). In der Alltagspraxis wird also ein Problem wahrgenommen, allerdings ohne es auf der Basis gewohnter Routinen genauer benennen zu können oder gar über Problemlösungen zu verfügen.

Mit der Formulierung der Fragestellungen durch die schulischen Akteure geht einher, dass sie auch die Reichweite des Problems bestimmen, quasi die Brennweite des Zooms, mit dem das Problem in den Blick genommen wird, bzw. den Grad der Grundsätzlichkeit, mit der das Problem bearbeitet wird. Dies immer wieder angemessen (anschlussfähig) auszubalancieren, stellt eine Herausforderung für die Wissenschaftlerinnen dar; wahrscheinlich handelt es sich hier um eine entscheidende Voraussetzung für das Gelingen bzw. Misslingen von Rückmeldegesprächen. Im Fall der Evaluation des Projekts „Jung \& Alt“ bezogen sich die Rekonstruktionsergebnisse auf relevante organisatorische und inhaltliche Teilaspekte des Projekts, die es in seiner Sinnhaftigkeit aber nicht grundsätzlich in Frage stellten. Vielmehr zeigte sich in der Analyse der Gruppendiskussionen auch, dass sich in dem Projekt intergenerationelle Lernprozesse ereignen und dass die Schüler*innen Fremdheitserfahrungen im Umgang mit den älteren Menschen als Herausforderungen bewältigen können und dabei Selbstwirksamkeit erfahren.

(2) Variation und Selektion: Mit der Rekonstruktion des konjunktiven, praktischen Handlungswissens der Akteure bezieht sich die Forschung auf die Handlungspraxis und stellt, indem das implizite Wissen begrifflich expliziert und der Reflexion zugänglich gemacht wird, Reflexionswissen für in der Praxis bereits bekannte Handlungsprobleme zur Verfügung. Damit werden die Lehrpersonen unterstützt, selbst

3 Hier besteht ein wesentlicher Unterschied zu dem weitverbreiteten Ansatz, Innovationen in Schulen durch die Implementation von Reformen oder Programmen und Instrumenten der Schul- und Unterrichtsentwicklung zu bewirken. Bei diesen Initiativen handelt es sich immer um Irritationen in der Umwelt des Systems der Einzelschule; es muss also damit gerechnet werden, dass sie durch das schulische System als nicht relevant angesehen oder in einer anderen als der intendierten Form umgesetzt werden, ergo scheitern können (vgl. Asbrand, 2013). 
eine Haltung der Beobachtung zweiter Ordnung einzunehmen und ihre Handlungsmöglichkeiten $\mathrm{zu}$ erweitern. Somit erzeugt die Forschung Variationen, die an die Handlungspraxis der Schule und die dort identifizierten Probleme anschlussfähig sind. ${ }^{4}$

Veränderung in der Organisation Schule entsteht im Modus der internen Komplexitätssteigerung und auf der Basis einer hohen Irritationsfähigkeit des Systems und nicht durch Reaktionen auf Impulse in der Umwelt (vgl. Luhmann, 2000, S. 74 f.). Damit ist die zweite Stellschraube für das Gelingen bzw. Misslingen responsiver Evaluationsprozesse angesprochen: Die Schule als lernende Organisation setzt voraus, dass ihre Mitglieder Veränderungen im Modus der Reflexion voranbringen (vgl. ebd., S. 47 ff.). Eine Gelingensbedingung scheint zu sein, Lehrpersonen als Professionelle $\mathrm{zu}$ adressieren und sich als Wissenschaftlerin mit wissenschaftlich fundierten Empfehlungen oder konzeptionellen Vorschlägen zurückzuhalten, sondern das Feld der Veränderung der schulischen Praxis den Lehrpersonen als Expert ${ }^{\star}$ innen für Schulentwicklung zu überlassen. In unserem Beispiel wurden durch die Evaluation zwar bestehende Strukturen grundsätzlich in Frage gestellt und die Irritationsfähigkeit der schulischen Akteure auf die Probe gestellt (die Frage, ob das Projekt überhaupt weitergeführt werden solle, stand im Raum); gleichzeitig waren einige Befunde aus den Analysen der Wissenschaftlerinnen für die Mitglieder der Fachkonferenz Religion offensichtlich aufschlussreich und solchermaßen bedenkenswert, so dass einzelne, aber wesentliche konzeptionelle Bestandteile des Projekts nachjustiert und erfolgreich verändert wurden (s. o.).

Schulentwicklung als evolutionären Prozess der Variation und Selektion zu verstehen, der sich innerhalb des schulischen Systems ereignet, bedeutet auch, als Wissenschaftlerin jederzeit mit dem Scheitern der wissenschaftlichen Begleitung zu rechnen. Zum einen können Rückmeldegespräche von den Lehrpersonen als nicht responsiv und die Forscherin als eine Person wahrgenommen werden, die den schulischen Akteuren „gute Ratschläge“ im Sinne rezeptartiger Handlungsvorschläge gibt. Zum anderen kann es passieren, dass Rekonstruktionsergebnisse, die im Wissenschaftssystem entstanden sind, in der schulischen Praxis nicht anschlussfähig sind. Nicht jede Rückmeldung von Forschungsergebnissen führt $\mathrm{zu}$ Veränderungen in der schulischen Praxis. In diesem Fall ist es notwendig (und zugleich herausfordernd), sich als Wissenschaftlerin auf die Systemreferenz der Wissenschaft zu besinnen und sich nicht von den Verbesserungs- und Fortschrittssemantiken des Schulentwicklungsdiskurses verleiten zu lassen. Die Güte der Forschung ist nicht da-

4 Im Unterschied zur Praxisforschung (vgl. z. B. Altrichter \& Feindt, 2011; Hahn, Heinrich \& Klewin, 2014) wird Praxisnähe in unserem Forschungszugang also nicht dadurch gewährleistet, dass die Lehrpersonen selbst forschen bzw. an der Datenerhebung und -analyse beteiligt werden. Dokumentarische Evaluationsforschung stellt Praxisnähe durch die Fundierung der Rekonstruktionen in der Alltagspraxis der Erforschten und durch die Responsivität des Evaluationsprozesses her (vgl. Bohnsack, 2006). 
von abhängig, dass ihre Ergebnisse in der Praxis als relevante Impulse bewertet werden und Innovationen bewirken.

(3) Restabilisierung: Nun kommt ins Spiel, was üblicherweise als Schulentwicklung verstanden wird: Kooperation in der Fachkonferenz und in Jahrgangsteams, ko-konstruktive Erarbeitung bzw. Überarbeitung von pädagogisch-didaktischen Konzepten, Verständigung über Ziele, die Schaffung von Strukturen innerhalb der Schule, die es ermöglichen, Erfahrungen innerhalb des Kollegiums zu teilen, die Initiierung einer Folge-Evaluation nach Überarbeitung und Erprobung des neuen Konzepts im Rahmen des schulinternen Qualitätsmanagements. All dies führt im Fall der HeleneLange-Schule dazu, dass die konzeptionellen Erneuerungen der außerunterrichtlichen Projekte in der Schule nachhaltig verankert werden (s.o.). Im Gegensatz zu einem Verständnis von Schulentwicklung als geplanter Entwicklung ist allerdings auffällig, dass die Instrumente der Schulentwicklung erst am Ende bzw. im Verlauf des Veränderungsprozesses relevant werden. Schulentwicklungsinstrumente scheinen hilfreich, um evolutionäre Prozesse zu unterstützen; ob sie sie auch initiieren können, bleibt eine offene Frage. Die organisationalen Strukturen der Schule bieten aber einen Rahmen für die Emergenz und Restabilisierung von Innovationen, einen Möglichkeitsraum für Veränderung.

\section{Literatur und Internetquellen}

Altrichter, H., \& Feindt, A. (2011). Lehrerinnen und Lehrer erforschen ihren Unterricht: Aktionsforschung. In E. Terhart, H. Bennewitz \& M. Rothland (Hrsg.), Handbuch der Forschung zum Lehrerberuf (S. 214-231). Münster: Waxmann.

Asbrand, B. (2013). Die dokumentarische Methode in der Governance-Forschung: Zur Rekonstruktion von Rekontextualisierungsprozessen. In K. Maag Merki, R. Langer \& H. Altrichter (Hrsg.), Educational Governance als Forschungsperspektive. Strategien, Methoden, Forschungsansätze (S. 177-198). Wiesbaden: VS. doi.org/10.1007/978-3-65806443-3_8

Bohnsack, R. (2006). Qualitative Evaluation und Handlungspraxis - Grundlagen dokumentarischer Evaluationsforschung. In U. Flick (Hrsg.), Qualitative Evaluationsforschung. Konzepte, Methoden, Umsetzungen (S. 135-155). Reinbek b. Hamburg: Rowohlt.

Bohnsack, R. (2014). Rekonstruktive Sozialforschung. Einführung in qualitative Methoden (9., überarb. und erweiterte Aufl.). Opladen: Barbara Budrich.

Bohnsack, R., \& Nentwig-Gesemann, I. (Hrsg.). (2010). Dokumentarische Evaluationsforschung. Theoretische Grundlagen und Beispiele aus der Praxis. Opladen: Barbara Budrich.

Emmerich, M., \& Maag Merki, K. (2014). Die Entwicklung von Schule. Theorie Forschung - Praxis. Enzyklopädie Erziehungswissenschaft Online. Weinheim: Beltz. doi. org/10.3262/EEO20140338

Hahn, S., Heinrich, M., \& Klewin, G. (2014). Forschung am Oberstufenkolleg - Bestandsaufnahme und Ausblick. In S. Hahn, M. Heinrich \& G. Klewin (Hrsg.), Forschung und Entwicklung am Oberstufen-Kolleg. Rückblick - Bestandsaufnahme - Ausblick (S. 81116). Münster: MV. 
John, R. (2013). Innovation als soziales Phänomen. In M. Rürup \& I. Bormann (Hrsg.), Innovationen im Bildungswesen. Analytische Zugänge und empirische Befunde (S. 7186). Wiesbaden: Springer VS. doi.org/10.1007/978-3-531-19701-2_3

Lamprecht, J. (2012). Rekonstruktiv-responsive Evaluation in der Praxis. Neue Perspektiven dokumentarischer Evaluationsforschung. Wiesbaden: VS. doi.org/10.1007/978-3-53193343-6

Luhmann, N. (1998). Die Gesellschaft der Gesellschaft. Frankfurt a. M.: Suhrkamp.

Luhmann, N. (2000). Organisation und Entscheidung. Opladen: Westdeutscher Verlag. doi. org/10.1007/978-3-322-97093-0

Mannheim, K. (1980). Strukturen des Denkens. Frankfurt a. M.: Suhrkamp.

Rosenberger, D. (in Vorb.). Außerunterrichtliche Aktivitäten aus der Sicht von Jugendlichen. Dissertation in Vorb. Goethe-Universität Frankfurt am Main.

Barbara Asbrand, Prof. Dr., geb. 1967, Professorin für Erziehungswissenschaft mit dem Schwerpunkt Allgemeine Didaktik und Schulentwicklung an der GoetheUniversität Frankfurt am Main.

Anschrift: Goethe-Universität Frankfurt am Main, Fachbereich Erziehungswissenschaften, Theodor-W.-Adorno-Platz 6, 60323 Frankfurt am Main

E-Mail: b.asbrand@em.uni-frankfurt.de

Carmen Bietz, geb. 1971, Direktorin als ständige Vertreterin des Leiters einer Gesamtschule an der Helene-Lange-Schule (IGS).

Anschrift: Helene-Lange-Schule, Langenbeckstr. 6-18, 65189 Wiesbaden

E-Mail: c.bietz@helene-lange-schule.de 